\title{
Análisis de Vibración de Platos Anulares con Soporte Elástico
}

\section{Vibration Analysis of Annular Plates with Elastic Support}

\section{José Alfredo Ramírez-Monares ${ }^{1}$}

1 Technische Universität Darmstadt

\section{RESUMEN}

La presente investigación consiste en un modelo mecánico de sujeción por resortes a flexión y a tensión-compresión para analizar el cambio en las frecuencias naturales y formas modales con respecto a las rigideces en la sujeción del disco. Las sujeciones empotradas de un sistema mecánico real son comúnmente modeladas como una sujeción ideal que restringe totalmente los momentos y fuerzas, lo cual comúnmente no existe en los sistemas reales. Esta limitación motiva el desarrollo del presente modelo que permite tomar en cuenta la elasticidad de la sujeción que existe en el disco anular. Se modela la vibración libre de un plato anular con aristas externa libre y diferentes valores de rigidez como condiciones de frontera en la arista interna y se resuelve analíticamente mediante las funciones de Bessel. Los parámetros de frecuencia natural $\gamma^{2}$ son tabulados para el soporte empotrado y elástico con diferentes relaciones de radios externo-interno, modos de vibración y rigideces en la arista interna. Se observa que conforme las rigideces del soporte flexible aumentan, los valores de los parámetros $\gamma^{2}$ convergen a los del disco con soporte clásico empotrado. Las formas modales cambian de manera distinta con respecto a los cambios en la rigidez a tensión-compresión y con respecto a cambios en la rigidez a flexión.

PALABRAS CLAVE: frecuencias naturales; disco anular; vibración libre; formas modales.

\section{ABSTRACT}

This research consists of a mechanical model of bending and tension-compression springs to analyze the change in natural frequencies and modal shapes with respect to the rigidities in the supported disc. The clamped fastening of a real mechanical system is commonly modeled as an ideal fastening that totally restricts the moments and forces, which commonly does not exist in real systems. This limitation motivates the development of the present model that allows considering the elasticity of the fastening that exists in the annular disc. The free vibration of an annular plate with outer free edge is modeled by different stiffness boundary conditions at the inner edge and solved analytically by the Bessel functions. The natural frequency parameters $\gamma^{2}$ are tabulated for the clamped and the elastic support for different internal-external radius ratios, modes of vibration and stiffnesses at the inner edge. It is observed that as the rigidities of the flexible support increase, the values of the parameters $\gamma^{2}$ converge to the classical clamped supported disk. Modal shapes change differently with respect to changes in stress-compression stiffness and with respect to changes in flexural stiffness.

KEYWORDS: natural frequency; annular plate; free vibration; modal shapes.

Correspondencia:

DESTINATARIO: José Alfredo Ramírez Monares INSTITUCIÓN: Technische Universität Darmstadt (Universidad Técnica de Darmstadt)

DIRECCIÓN: Dolivostr. 15, 64293, Darmstadt, Alemania CORREO ELECTRÓNICO: ramirez@dyn.tu-darmstadt.de
Fecha de recepción: 9 de noviembre de 2020. Fecha de aceptación: 16 de diciembre de 2020 . Fecha de publicación: 22 de diciembre de 2020.
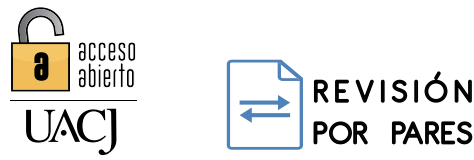

Licencia Creative Commons

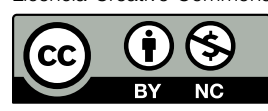




\section{INTRODUCCIÓN}

La naturaleza del tipo de sujeción de las estructuras conduce al uso de condiciones de frontera como apoyos simples o empotrados, en cuyo caso, se supone que los desplazamientos o pendientes cero se mantienen en los límites. Sin embargo, en aplicaciones físicas, es imposible mantener condiciones de frontera realmente fijas. Esto puede deberse a propiedades físicas del sistema que se está modelando, limitaciones en los mecanismos de sujeción o propiedades del material que impiden que las abrazaderas sean perfectas, por ejemplo, se producirá un deslizamiento si el material de la estructura que se sujeta es significativamente más blando que las abrazaderas.

Una forma en la que se manifiesta la pérdida de rigidez debida al movimiento en las condiciones de contorno es a través de una disminución de las frecuencias naturales de la estructura o sistema acústico estructural. Este cambio en las frecuencias puede variar desde niveles de 1-2 \% en sistemas bien sujetos a $20 \%-30 \%$ en sistemas con sujeción débil ${ }^{[1]}$. Sin embargo, estos sistemas de sujeción floja aun soportan un momento, de modo que las condiciones de frontera de soportes simples o empotrados (desplazamiento cero y momento cero) no son apropiadas.

Para representar tales fenómenos el mecanismo de sujeción de un disco anular se modela como un resorte que opone resistencia a la flexión y un resorte que se opone al desplazamiento vertical en la ubicación del mecanismo de sujeción. En el presente estudio se considera el modelado de su sujeción bajo vibración libre mediante la teoría de placas delgadas de Kirchhoff.

En los trabajos de Reddy [2] y Weaver [3] se muestran funciones de desplazamiento de placas anulares con diversas condiciones de frontera y diferentes tipos de cargas distribuidas y puntuales. Ellos obtienen soluciones utilizando funciones de Bessel y el método de Rayleigh-Ritz. En el trabajo de Wang ${ }^{[4]}$ se analizan discos circulares con soportes elásticos distribuidos en un área del disco y diversas condiciones de frontera en la arista externa. Leissa ${ }^{[5]}$ presenta soluciones analíticas para el análisis de vibración libre de placas anulares isotrópicas delgadas que tienen diferentes condiciones de soporte. Sin embargo, en estos trabajos no existen estrategias claramente definidas para las sujeciones no ideales que existen en los sistemas mecánicos reales, tales como la elasticidad en algunos soportes.
En ${ }^{[6]}$ y ${ }^{[7]}$ también se presentan soluciones analíticas para discos con soportes flexibles distribuidos en una región del disco circular, sin embargo, estas soluciones son funciones definidas por tramos cuya localización de raíces es complicada y tediosa, además de que solo incluyen una rigidez distribuida en un área del disco. En el trabajo de Laura ${ }^{[8]}$ se estudian las vibraciones libres con el método de Rayleigh-Ritz en un disco circular con cambio lineal de grosor en la dirección radial, también considerando una rigidez distribuida en solo un área del disco. En ${ }^{[9]}$ y ${ }^{[10]}$ se estudian discos circulares con cambio de grosor, en ${ }^{[9]}$ se incluyen los efectos por el incremento en la temperatura y en ${ }^{[10]}$ se presenta un disco con dos soportes, uno de ellos con rigideces a flexión y a desplazamiento vertical similar al que se presenta aquí, sin embargo, en el presente trabajo se modela este tipo de soporte en la arista interior de un disco anular sin cambios en el grosor. En ${ }^{[11]}$ se estudian algunos modelos de placas con geometrías de sectores de disco, anulares y circulares, todas ellas compuestas laminadas, es decir, formadas por capas con diferentes propiedades. En los resultados reportados en [11] se menciona que al incrementar la rigidez los valores obtenidos del parámetro de frecuencia natural se asemejan a los correspondientes de un soporte rígido.

\section{METODOLOGÍA}

El presente estudio hace uso de la teoría de placas delgadas de Kirchhoff para la solución en vibración libre de discos anulares con soporte elástico. La metodología del presente trabajo de investigación es:

1. El establecimiento de las ecuaciones que definen las condiciones de frontera para los modelos de soporte empotrado y soporte elástico.

2. La solución de la matriz característica y la obtención de los parámetros de frecuencia natural.

3. La presentación de los resultados en forma tabular para las diversas combinaciones de rigidez, diámetros del disco anular y modos de vibración.

4. El análisis de los parámetros de frecuencia natural con respecto a los cambios en las rigideces.

5. El análisis modal del disco para cambios en una rigidez, manteniendo la otra constante. 


\section{Modelo Clásico de Disco Anular con Aristas Externa Libre e Interna Empotrada}

El modelo mecánico más simple considera la placa circular como una placa anular con la arista interna empotrada, lo cual está representado en la Figura 1. De acuerdo con [12], la ecuación diferencial de movimiento, considerando las energías cinética y de deformación por flexión, es como se muestra en la Ecuación (1).

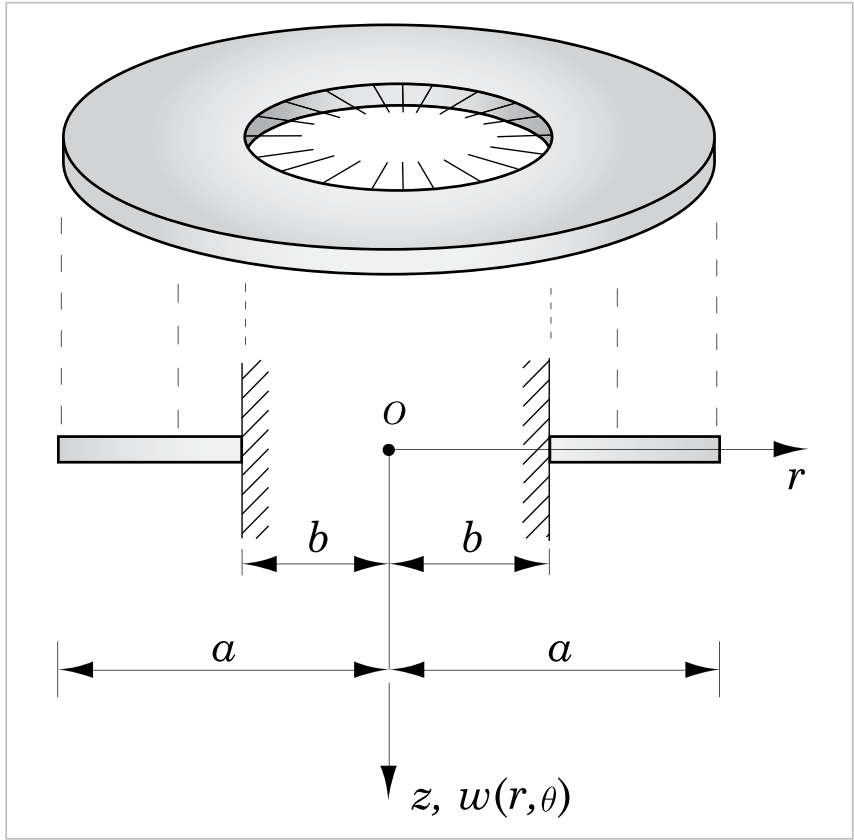

Figura 1. Plato anular con aristas externa libre e interna empotrada.

$$
\left(\nabla^{2}+\gamma^{2}\right)\left(\nabla^{2}-\gamma^{2}\right) w=0
$$

La forma general de desplazamiento lateral de vibración de la placa se puede expresar como se muestra en la Ecuación (2).

$$
\begin{aligned}
w(r, \theta)=\sum_{m=0}^{\infty} R_{m, n}(\gamma r) \cos (m \theta) & \\
& +\sum_{m=1}^{\infty} R_{m, n}(\gamma r) \operatorname{sen}(m \theta)
\end{aligned}
$$

donde $(r, \theta)$ son coordenadas polares, $m$ y $n$ son el número de diámetros y círculos modales respectivamente, $\gamma_{m, n}^{4}=a^{2} \omega^{2}(\rho h / D)^{1 / 2}$ es el parámetro adimensional de la frecuencia natural, $D=E h^{3} / 12$ es la constante de rigidez a flexión, $R$ es una combinación lineal de las funciones de Bessel $J_{m}(\gamma r), Y_{m}(\gamma r), I_{m}(\gamma r)$ y $K_{m}(\gamma r)$, donde $J_{m}(\gamma r)$ es la función de Bessel del primer tipo, $Y_{m}(\gamma r)$ es la función de Bessel del segundo tipo, $I_{m}(\gamma r)$ es la función de Bessel modificada del primer tipo y $K_{m}(\gamma r)$ es la función de
Bessel modificada del segundo tipo. La solución para este modelo mecánico es una función combinada en la dirección radial que se muestra en la Ecuación (3).

$$
\begin{aligned}
R_{n}=C_{1} J_{m}(\gamma r)+ & C_{2} Y_{m}(\gamma r)+C_{3} I_{m}(\gamma r) \\
& +C_{4} K_{m}(\gamma r) \\
b \leq & r \leq a
\end{aligned}
$$

Y las condiciones de frontera son las que se muestran en las Ecuaciones (4) a (7).

$$
\begin{gathered}
w(b, \theta, t)=0 \Rightarrow R_{m}(b)=0 \\
w_{r r}(b, \theta, t)=0 \Rightarrow R_{m}^{\prime}(b)=0 \\
\begin{aligned}
V_{r}(a, \theta)=0 \Rightarrow Q_{r}(a, \theta)-\frac{1}{a} \frac{\partial M_{r t}}{\partial \theta}(a, \theta) \\
=0
\end{aligned} \\
M_{r}(a, \theta)=0 \Rightarrow \frac{\partial^{2} w}{\partial r^{2}}(a, \theta) \\
+v\left[\frac{1}{a} \frac{\partial w}{\partial r}(a, \theta)\right. \\
\left.+\frac{1}{a^{2}} \frac{\partial^{2} w}{\partial \theta^{2}}(a, \theta)\right]=0
\end{gathered}
$$

donde $w_{r r}$ es la derivada de la función de desplazamiento $w$ con respecto al radio $r, M_{r}$ es el momento flector, $v$ es el coeficiente de Poisson, $V_{r}$ es el esfuerzo cortante, $Q_{r}$ es la fuerza cortante y $M_{r t}$ es el momento de torsión. Entonces, de acuerdo con ${ }^{[7]}$, la matriz característica se define en la Ecuación (8):

$$
\left[\begin{array}{cccc}
J_{m}(\gamma b) & Y_{m}(\gamma b) & I_{m}(\gamma b) & K_{m}(\gamma b) \\
R_{m 1}^{\prime} & R_{m 2}^{\prime} & R_{m 3}^{\prime} & R_{m 4}^{\prime} \\
V_{r 1}^{\prime} & V_{r 2} & V_{r 3} & V_{r 4} \\
M_{r 1} & M_{r 2} & M_{r 3} & M_{r 4}
\end{array}\right]\left\{\begin{array}{c}
C_{1} \\
C_{2} \\
C_{3} \\
C_{4}
\end{array}\right\}=\left\{\begin{array}{l}
0 \\
0 \\
0 \\
0
\end{array}\right\}
$$

donde

$$
\begin{aligned}
R_{m 1}^{\prime} & =-\gamma J_{m+1}(\gamma b)+\frac{m}{r} J_{m}(\gamma b) \\
R_{m 2}^{\prime} & =-\gamma Y_{m+1}(\gamma b)+\frac{m}{r} Y_{m}(\gamma b) \\
R_{m 3}^{\prime} & =\gamma I_{m+1}(\gamma b)+\frac{m}{r} I_{m}(\gamma b) \\
R_{m 4}^{\prime} & =\gamma K_{m+1}(\gamma b)+\frac{m}{r} K_{m}(\gamma b)
\end{aligned}
$$




$$
\begin{aligned}
& V_{r 1}=m J_{m}(\gamma a)-\gamma a J_{m+1}(\gamma a) \\
& +\frac{m^{2}(1-v)}{\gamma^{2} a^{2}}[(m \\
& \text {-1)J } J_{m}(\gamma a) \\
& \left.-\gamma a J_{m+1}(\gamma a)\right] \\
& V_{r 2}=m Y_{m}(\gamma a)-\gamma a Y_{m+1}(\gamma a) \\
& +\frac{m^{2}(1-v)}{\gamma^{2} a^{2}}[(m \\
& \text {-1) } Y_{m}(\gamma a) \\
& \left.-\gamma a Y_{m+1}(\gamma a)\right] \\
& V_{r 3}=-m I_{m}(\gamma a)-\gamma a I_{m+1}(\gamma a) \\
& +\frac{m^{2}(1-v)}{\gamma^{2} a^{2}}[(m \\
& \text {-1) } \left.I_{m}(\gamma a)+\gamma a I(\gamma a)\right] \\
& V_{r 4}=-m K_{m}(\gamma a)+\gamma a K_{m+1}(\gamma a) \\
& +\frac{m^{2}(1-v)}{\gamma^{2} a^{2}}[(m \\
& \text {-1) } K_{m}(\gamma a) \\
& \left.-\gamma a K_{m+1}(\gamma a)\right] \\
& M_{r 1}=J_{m}(\gamma a)-(1-v)\left[\frac{m(m-1)}{\gamma^{2} a^{2}} J_{m}(\gamma a)\right. \\
& \left.+\frac{1}{\gamma a} J_{m+1}(\gamma a)\right] \\
& M_{r 2}=Y_{m}(\gamma a)-(1-v)\left[\frac{m(m-1)}{\gamma^{2} a^{2}} Y_{m}(\gamma a)\right. \\
& \left.+\frac{1}{\gamma a} Y_{m+1}(\gamma a)\right] \\
& M_{r 3}=-I_{m}(\gamma a)-(1-v) \frac{m(m-1)}{\gamma^{2} a^{2}} I_{m} \gamma a \\
& -\frac{1}{\gamma a} I_{m+1}(\gamma a) \\
& M_{r 4}=-K_{m}(\gamma a)-(1 \\
& -v)\left[\frac{m(m-1)}{\gamma^{2} a^{2}} K_{m}(\gamma a)\right. \\
& \left.+\frac{1}{\gamma a} K_{m+1}(\gamma a)\right]
\end{aligned}
$$

Ahora se pueden encontrar los parámetros de las frecuencias naturales $\gamma_{m, n}^{2}$ y las formas modales obtenidas a partir de las constantes $C_{1}$ a $C_{4}$ de la Ecuación (8).

\section{Modelo de Disco Anular con Soporte Elástico}

Ahora se considera el disco anular de radio exterior $a$ con un apoyo elástico formado por resortes distribuidos uniformemente alrededor de su borde interior, como se muestra en la Figura 2. La solución en dirección radial es la misma que se expone en la Ecuación (2). Se tienen los resortes de rigidez distribuida $k_{w}$ que se opone a la traslación en la dirección $z$ en todo el contorno de radio $b$, y la rigidez distribuida $k_{\psi}$ que se opone a la flexión en todo el contorno de radio $b$. Las condiciones de frontera relativas a la fuerza cortante y momento flexionante de las Ecuaciones (4) y (5) cambian por las que se presentan en las Ecuaciones (21) y (22).

$$
\begin{aligned}
V_{r}(b, \theta)=-k_{w} w & (b, \theta) \\
& \Rightarrow Q_{r}(b, \theta) \\
& -\frac{1}{b} \frac{\partial M_{r t}}{\partial \theta}(b, \theta) \\
& =-k_{w} w(b, \theta) \\
M_{r}(b, \theta)=k_{\psi} w_{r r}( & b, \theta) \\
& \Rightarrow \frac{\partial^{2} w}{\partial r^{2}}(b, \theta) \\
& +v\left[\frac{1}{b} \frac{\partial w}{\partial r}(b, \theta)\right. \\
& \left.+\frac{1}{b^{2}} \frac{\partial^{2} w}{\partial \theta^{2}}(b, \theta)\right] \\
& =k_{\psi} \frac{\partial w}{\partial r}(b, \theta)
\end{aligned}
$$

Las condiciones de frontera referentes al contorno libre que se muestran en las Ecuaciones (6) y (7) permanecen válidas.

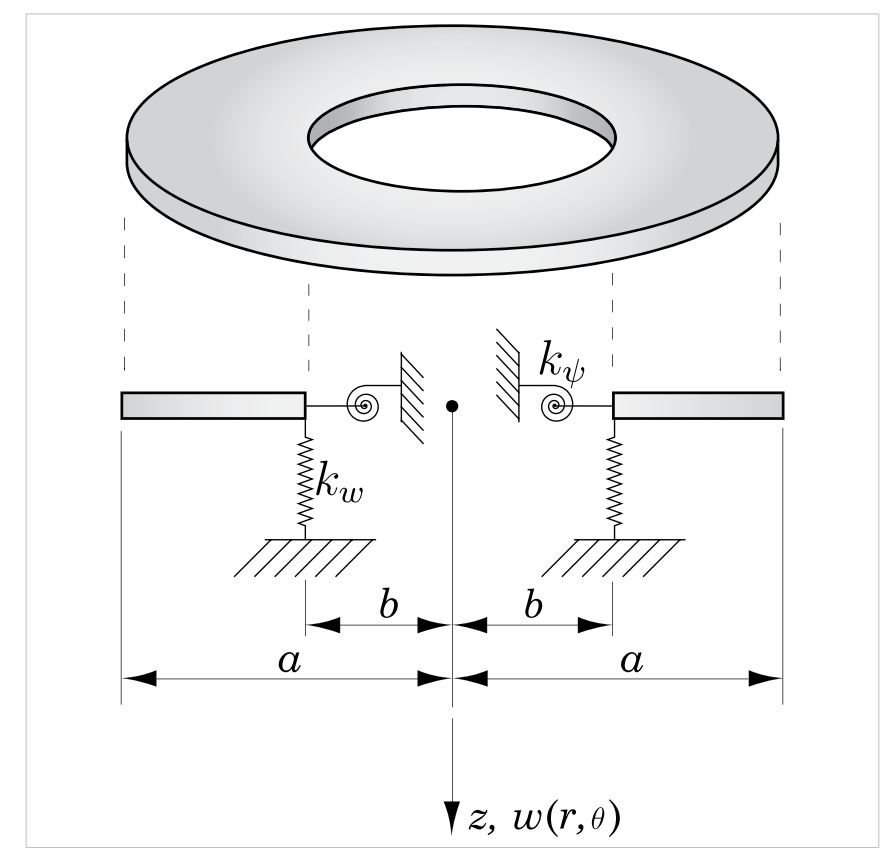

Figura 2. Plato anular con aristas externa libre e interna con soporte elástico. 


\section{RESULTADOS}

La Tabla 1 muestra los valores de los parámetros adimensionales de las frecuencias naturales $\gamma_{m, n}^{2}$ para diferentes relaciones de radio interno $b$-radio externo $a$, diámetros $m$ y círculos $n$ modales obtenidos aquí y por las referencias para el modelo clásico de disco anular con aristas externa libre interna empotrada. Debajo de cada parámetro está el valor en porcentaje de la diferencia entre los valores de las referencias y los del presente trabajo. En todos los casos de esta Tabla 1 se observa una similitud aceptable (\%) entre los resultados aquí obtenidos [Pres.] y los de las referencias.

TABLA 1

Parámetros Adimensionales de las Frecuencias Naturales $\gamma_{m, n}^{2}$ Para Disco Anular con Arista Externa libre e Interna EMPOTRADA

\begin{tabular}{|c|c|c|c|c|c|c|c|}
\hline \multirow{2}{*}{$m$} & \multirow{2}{*}{$n$} & \multirow{2}{*}{ [REF.] } & \multicolumn{5}{|c|}{$m$} \\
\hline & & & 0.1 & 0.3 & 0.5 & 0.7 & 0.9 \\
\hline \multirow{11}{*}{0} & \multirow{11}{*}{1} & Pres. & 4.262 & 6.701 & 13.089 & 37.069 & 344.761 \\
\hline & & [13] & 4.237 & 6.66 & 13.024 & 36.953 & 345 \\
\hline & & $\%$ & 0.586 & 0.611 & 0.4966 & 0.3129 & 0.069 \\
\hline & & [5] & 4.235 & 6.739 & 13.05 & 36.6 & --. \\
\hline & & $\%$ & 0.6375 & 0.567 & 0.2979 & 1.2652 & \\
\hline & & [14] & -- & -- & 13.1 & -- & |-- \\
\hline & & $\%$ & & & 0.084 & & \\
\hline & & [15] & 4.23 & 6.66 & 13.0 & 37 & |-- \\
\hline & & $\%$ & 0.7565 & 0.6118 & 0.6799 & 0.1861 & \\
\hline & & [7] & -- & 6.66 & 13.024 & 36.953 & -- \\
\hline & & $\%$ & & 0.6118 & 0.4966 & 0.3129 & \\
\hline \multirow{7}{*}{0} & \multirow{7}{*}{2} & Pres. & 25.346 & 42.721 & 85.1793 & 240.081 & 2189.52 \\
\hline & & [1] & 25.262 & 42.614 & 85.032 & -- & -- \\
\hline & & $\%$ & 0.3314 & 0.2504 & 0.1729 & & \\
\hline & & [3] & 25.3 & 42.6 & 85.1 & 239 & -- \\
\hline & & $\%$ & 0.1814 & 0.2832 & 0.093 & 0.4502 & \\
\hline & & [7] & 25.3 & 42.6 & 85.1 & 239 & -- \\
\hline & & $\%$ & 0.1814 & 0.2832 & 0.093 & 0.4502 & \\
\hline \multirow{7}{*}{1} & \multirow{7}{*}{1} & Pres. & 3.479 & 6.56 & 13.313 & 37.566 & 345.743 \\
\hline & & [5] & 3.482 & -- & 13.3 & 37.498 & 345.0 \\
\hline & & $\%$ & 0.0862 & & 0.0976 & 0.181 & 0.2148 \\
\hline & & [7] & -- & -- & 13.3 & -- & -- \\
\hline & & $\%$ & & & 0.0976 & & \\
\hline & & [15] & -- & 6.552 & 13.29 & 37.5 & -- \\
\hline & & $\%$ & & 0.1219 & 0.1727 & 0.1756 & \\
\hline \multirow{3}{*}{1} & \multirow{3}{*}{2} & Pres. & 27.725 & 44.7059 & 86.82 & 241.489 & 2190.77 \\
\hline & & [5] & 27.3 & 44.6 & 86.7 & 241 & 2189 \\
\hline & & $\%$ & 1.5329 & 0.2368 & 0.1382 & 0.2024 & 0.08 \\
\hline
\end{tabular}

En las Tablas 2 y 3 se muestran los parámetros adimensionales de las frecuencias naturales $\gamma_{0,1}^{2}$ y $\gamma_{0,2}^{2}$ para diferentes combinaciones de rigideces $k_{w}$ y $k_{\psi}$ y en el disco anular con relación $b / a=0.1$. (De las Tablas 2 a la 21 , los datos corresponden al disco anular con arista externa libre e interna soportada por resortes $k_{w} \mathrm{y} k_{\psi}$ ).

TABLA 2

Parámetros Adimensionales de las Frecuencias NATURALES $\gamma_{0,1}^{2}, b / a=0.1$

\begin{tabular}{|c|c|c|c|c|c|c|}
\hline \multirow{2}{*}{$\begin{array}{c}k_{w}(\mathrm{~N} / \mathrm{m} \\
\left.10^{2}\right)\end{array}$} & \multicolumn{6}{|c|}{$k_{\psi}\left(\mathrm{Nm} 10^{2}\right)$} \\
\cline { 2 - 7 } & 0.1 & 0.25 & 0.5 & 1 & 10 & 100 \\
\hline 0.1 & 5.2277 & 4.8105 & 4.7124 & 4.6689 & 4.6326 & 4.6291 \\
\hline 0.25 & 4.9926 & 4.5844 & 4.4899 & 4.4482 & 4.4133 & 4.4100 \\
\hline 0.5 & 4.9128 & 4.5084 & 4.4152 & 4.3742 & 4.3399 & 4.3366 \\
\hline 1 & 4.8726 & 4.4703 & 4.3779 & 4.3372 & 4.3032 & 4.3000 \\
\hline 10 & 4.8364 & 4.4360 & 4.3443 & 4.3039 & 4.2702 & 4.2670 \\
\hline 100 & 4.8328 & 4.4326 & 4.3409 & 4.3005 & 4.2669 & 4.2637 \\
\hline
\end{tabular}

TABLA 3

Parámetros Adimensionales de las Frecuencias NATURALES $\gamma_{0,2}^{2}, b / a=0.1$

\begin{tabular}{|c|c|c|c|c|c|c|}
\hline \multirow{2}{*}{$\begin{array}{c}k_{w}(\mathrm{~N} / \mathrm{m} \\
\left.10^{2}\right)\end{array}$} & \multicolumn{6}{|c|}{$k_{\psi}\left(\mathrm{Nm} 10^{2}\right)$} \\
\cline { 2 - 7 } & 0.1 & 0.25 & 0.5 & 1 & 10 & 100 \\
\hline 0.1 & 28.8128 & 27.7664 & 27.4749 & 27.3383 & 27.2202 & 27.2087 \\
\hline 0.25 & 27.6502 & 26.6381 & 26.3598 & 26.2299 & 26.1179 & 26.1069 \\
\hline 0.5 & 27.2444 & 26.2488 & 25.9763 & 25.8493 & 25.7399 & 25.7292 \\
\hline 1 & 27.0385 & 26.0522 & 25.7829 & 25.6574 & 25.5493 & 25.5388 \\
\hline 10 & 26.8517 & 25.8743 & 25.6079 & 25.4839 & 25.3771 & 25.3667 \\
\hline 100 & 26.8329 & 25.8565 & 25.5904 & 25.4665 & 25.3599 & 25.3495 \\
\hline
\end{tabular}

En las Tablas 4 y 5 se muestran los parámetros adimensionales de las frecuencias naturales $\gamma_{1,1}^{2}$ y $\gamma_{1,2}^{2}$ para diferentes combinaciones de rigideces $k_{w} \mathrm{y} k_{\psi}$ en el disco anular con relación $b / a=0.1$.

TABLA 4

Parámetros Adimensionales de las Frecuencias NATURALES $\gamma_{1,1}^{2}, b / a=0.1$

\begin{tabular}{|c|c|c|c|c|c|c|}
\hline \multirow{2}{*}{$\begin{array}{c}k_{w}(\mathrm{~N} / \mathrm{m} \\
\left.10^{2}\right)\end{array}$} & \multicolumn{7}{|c|}{$k_{\psi}\left(\mathrm{Nm} 10^{2}\right)$} \\
\cline { 2 - 7 } & 0.1 & 0.25 & 0.5 & 1 & 10 & 100 \\
\hline 0.1 & 12.5707 & 12.5689 & 12.5685 & 12.5684 & 12.5682 & 12.5682 \\
\hline 0.25 & 6.8790 & 6.7123 & 6.6834 & 6.6715 & 6.6619 & 6.6609 \\
\hline 0.5 & 5.4030 & 4.8565 & 4.7640 & 4.7265 & 4.6967 & 4.6939 \\
\hline 1 & 5.0110 & 4.2256 & 4.0813 & 4.0221 & 3.9748 & 3.9703 \\
\hline 10 & 4.7988 & 3.8531 & 3.6667 & 3.5888 & 3.5260 & 3.5200 \\
\hline 100 & 4.7825 & 3.8242 & 3.6343 & 3.5548 & 3.4905 & 3.4844 \\
\hline
\end{tabular}


TABLA 5

Parámetros Adimensionales de las Frecuencias NATURALES $\gamma_{1,2}^{2}, b / a=0.1$

\begin{tabular}{|c|c|c|c|c|c|c|}
\hline \multirow{2}{*}{$\begin{array}{c}k_{w}(\mathrm{~N} / \mathrm{m} \\
10^{2}\end{array}$} & \multicolumn{6}{|c|}{$k_{\psi}\left(\mathrm{Nm} 10^{2}\right)$} \\
\cline { 2 - 7 } & 0.1 & 0.25 & 0.5 & 1 & 10 & 100 \\
\hline 0.1 & 34.5677 & 33.6762 & 33.4371 & 33.3264 & 33.2314 & 33.2221 \\
\hline 0.25 & 30.9772 & 29.9492 & 29.6816 & 29.5588 & 29.4540 & 29.4438 \\
\hline 0.5 & 30.0420 & 29.0092 & 28.7430 & 28.6212 & 28.5174 & 28.5073 \\
\hline 1 & 29.6261 & 28.5965 & 28.3322 & 28.2115 & 28.1087 & 28.0987 \\
\hline 10 & 29.2799 & 28.2553 & 27.9933 & 27.8738 & 27.7721 & 27.7622 \\
\hline 100 & 29.2466 & 28.2227 & 27.9610 & 27.8416 & 27.7400 & 27.7301 \\
\hline
\end{tabular}

En las Tablas 6 y 7 se muestran los parámetros adimensionales de las frecuencias naturales $\gamma_{0,1}^{2}$ y $\gamma_{0,2}^{2}$ para diferentes combinaciones de rigideces $k_{w}$ y $k_{\psi}$ en el disco anular con relación $b / a=0.3$.

TABLA 6

Parámetros Adimensionales de las Frecuencias NATURALES $\gamma_{0,1}^{2}, b / a=0.3$

\begin{tabular}{|c|c|c|c|c|c|c|}
\hline \multirow{2}{*}{$\begin{array}{c}k_{w}(\mathrm{~N} / \mathrm{m} \\
\left.10^{2}\right)\end{array}$} & \multicolumn{6}{|c|}{$k_{\psi}\left(\mathrm{Nm} 10^{2}\right)$} \\
\cline { 2 - 7 } & 0.1 & 0.25 & 0.5 & 1 & 10 & 100 \\
\hline 0.1 & 8.3651 & 7.7742 & 7.6011 & 7.5186 & 7.4465 & 7.4394 \\
\hline 0.25 & 7.8933 & 7.3230 & 7.1575 & 7.0789 & 7.0103 & 7.0036 \\
\hline 0.5 & 7.7276 & 7.1666 & 7.0043 & 6.9273 & 6.8602 & 6.8536 \\
\hline 1 & 7.6433 & 7.0874 & 6.9269 & 6.8508 & 6.7845 & 6.7779 \\
\hline 10 & 7.5667 & 7.0157 & 6.8569 & 6.7815 & 6.7160 & 6.7095 \\
\hline 100 & 7.5590 & 7.0086 & 6.8498 & 6.7746 & 6.7091 & 6.7027 \\
\hline
\end{tabular}

TABLA 7

Parámetros Adimensionales de las Frecuencias NATURALES $\gamma_{0,2}^{2}, b / a=0.3$

\begin{tabular}{|c|c|c|c|c|c|c|}
\hline \multirow{2}{*}{$\begin{array}{c}k_{w}(\mathrm{~N} / \mathrm{m} \\
\left.10^{2}\right)\end{array}$} & \multicolumn{6}{|c|}{$k_{\psi}\left(\mathrm{Nm} 10^{2}\right)$} \\
\cline { 2 - 7 } & 0.1 & 0.25 & 0.5 & 1 & 10 & 100 \\
\hline 0.1 & 50.2493 & 48.7577 & 48.3037 & 48.0842 & 47.8910 & 47.8719 \\
\hline 0.25 & 47.3355 & 45.8509 & 45.4061 & 45.1924 & 45.0048 & 44.9863 \\
\hline 0.5 & 46.1925 & 44.7314 & 44.2963 & 44.0877 & 43.9048 & 43.8867 \\
\hline 1 & 45.5884 & 44.1441 & 43.7155 & 43.5101 & 43.3303 & 43.3125 \\
\hline 10 & 45.0265 & 43.6007 & 43.1788 & 42.9768 & 42.8000 & 42.7826 \\
\hline 100 & 44.9694 & 43.5456 & 43.1244 & 42.9228 & 42.7464 & 42.7290 \\
\hline
\end{tabular}

En las Tablas 8 y 9 se muestran los parámetros adimensionales de las frecuencias naturales $\gamma_{1,1}^{2}$ y $\gamma_{1,2}^{2}$ para diferentes combinaciones de rigideces $k_{w} \mathrm{Y} k_{\psi}$ en el disco anular con relación $b / a=0.3$.
TABLA 8

Parámetros Adimensionales de las Frecuencias NATURALES $\gamma_{1,1}^{2}, b / a=0.3$

\begin{tabular}{|c|c|c|c|c|c|c|}
\hline \multirow{2}{*}{$\begin{array}{c}k_{w}(\mathrm{~N} / \mathrm{m} \\
\left.10^{2}\right)\end{array}$} & \multicolumn{7}{|c|}{$k_{\psi}\left(\mathrm{Nm} 10^{2}\right)$} \\
\cline { 2 - 7 } & 0.1 & 0.25 & 0.5 & 1 & 10 & 100 \\
\hline 0.1 & 9.4769 & 8.8517 & 8.6725 & 8.5878 & 8.5141 & 8.5069 \\
\hline 0.25 & 8.3069 & 7.6559 & 7.4716 & 7.3848 & 7.3096 & 7.3022 \\
\hline 0.5 & 7.9274 & 7.2748 & 7.0908 & 7.0042 & 6.9293 & 6.9219 \\
\hline 1 & 7.7426 & 7.0905 & 6.9070 & 6.8207 & 6.7460 & 6.7387 \\
\hline 10 & 7.5797 & 6.9289 & 6.7460 & 6.6601 & 6.5858 & 6.5785 \\
\hline 100 & 7.5636 & 6.9130 & 6.7302 & 6.6443 & 6.5700 & 6.5627 \\
\hline
\end{tabular}

TABLA 9

Parámetros Adimensionales de las Frecuencias NATURALES $\gamma_{1,2}^{2}, b / a=0.3$

\begin{tabular}{|c|c|c|c|c|c|c|}
\hline \multirow{2}{*}{$\begin{array}{c}k_{w}(\mathrm{~N} / \mathrm{m} \\
\left.10^{2}\right)\end{array}$} & \multicolumn{7}{|c|}{$k_{\psi}\left(\mathrm{Nm} 10^{2}\right)$} \\
\cline { 2 - 7 } & 0.1 & 0.25 & 0.5 & 1 & 10 & 100 \\
\hline 0.1 & 52.9231 & 51.4628 & 51.0206 & 50.8073 & 50.6197 & 50.6012 \\
\hline 0.25 & 49.5595 & 48.0997 & 47.6656 & 47.4576 & 47.2752 & 47.2573 \\
\hline 0.5 & 48.2615 & 46.8283 & 46.4051 & 46.2028 & 46.0257 & 46.0082 \\
\hline 1 & 47.5824 & 46.1689 & 45.7530 & 45.5544 & 45.3806 & 45.3635 \\
\hline 10 & 46.9558 & 45.5638 & 45.1556 & 44.9608 & 44.7905 & 44.7737 \\
\hline 100 & 46.8924 & 45.5028 & 45.0953 & 44.9009 & 44.7311 & 44.7143 \\
\hline
\end{tabular}

En las Tablas 10 y 11 se muestran los parámetros adimensionales de las frecuencias naturales $\gamma_{0,1}^{2}$ y $\gamma_{0,2}^{2}$ para diferentes combinaciones de rigideces $k_{w} \mathrm{y} k_{\psi}$ en el disco anular con relación $b / a=0.5$.

TABLA 10

Parámetros Adimensionales de las Frecuencias NATURALES $\gamma_{0,1}^{2}, b / a=0.5$

\begin{tabular}{|c|c|c|c|c|c|c|}
\hline \multirow{2}{*}{$\begin{array}{c}k_{w}(\mathrm{~N} / \mathrm{m} \\
\left.10^{2}\right)\end{array}$} & \multicolumn{7}{|c|}{$k_{\psi}\left(\mathrm{Nm} 10^{2}\right)$} \\
\cline { 2 - 7 } & 0.1 & 0.25 & 0.5 & 1 & 10 & 100 \\
\hline 0.1 & 16.9547 & 15.8085 & 15.4579 & 15.2882 & 15.1387 & 15.1239 \\
\hline 0.25 & 15.7190 & 14.6089 & 14.2735 & 14.1118 & 13.9697 & 13.9556 \\
\hline 0.5 & 15.2543 & 14.1672 & 13.8401 & 13.6826 & 13.5443 & 13.5306 \\
\hline 1 & 15.0126 & 13.9393 & 13.6170 & 13.4620 & 13.3259 & 13.3124 \\
\hline 10 & 14.7901 & 13.7306 & 13.4131 & 13.2604 & 13.1264 & 13.1132 \\
\hline 100 & 14.7676 & 13.7096 & 13.3925 & 13.2401 & 13.1063 & 13.0931 \\
\hline
\end{tabular}

TABLA 11

Parámetros Adimensionales de las Frecuencias NATURALES $\gamma_{0,2}^{2}, b / a=0.5$

\begin{tabular}{|c|c|c|c|c|c|c|}
\hline \multirow{2}{*}{$\begin{array}{c}k_{w}(\mathrm{~N} / \mathrm{m} \\
\left.10^{2}\right)\end{array}$} & \multicolumn{7}{|c|}{$k_{\psi}\left(\mathrm{Nm} 10^{2}\right)$} \\
\cline { 2 - 7 } & 0.1 & 0.25 & 0.5 & 1 & 10 & 100 \\
\hline 0.1 & 104.8303 & 102.2243 & 101.4100 & 101.0128 & 100.6609 & 100.6260 \\
\hline 0.25 & 97.3198 & 94.5348 & 93.6803 & 93.2662 & 92.9008 & 92.8647 \\
\hline 0.5 & 93.7362 & 90.9541 & 90.1085 & 89.7001 & 89.3405 & 89.3049 \\
\hline 1 & 91.6903 & 88.9359 & 88.1033 & 87.7019 & 87.3488 & 87.3140 \\
\hline 10 & 89.6975 & 86.9880 & 86.1731 & 85.7809 & 85.4363 & 85.4023 \\
\hline 100 & 89.4903 & 86.7864 & 85.9737 & 85.5826 & 85.2390 & 85.2050 \\
\hline
\end{tabular}


En las Tablas 12 y 13 se muestran los parámetros adimensionales de las frecuencias naturales $\gamma_{1,1}^{2}$ y $\gamma_{1,2}^{2}$ para diferentes combinaciones de rigideces $k_{w} \mathrm{y} k_{\psi}$ en el disco anular con relación $b / a=0.5$.

TABLA 12

Parámetros Adimensionales de las Frecuencias NATURALES $\gamma_{1,1}^{2}, b / a=0.5$

\begin{tabular}{|c|c|c|c|c|c|c|}
\hline \multirow{2}{*}{$\begin{array}{c}k_{w}(\mathrm{~N} / \mathrm{m} \\
\left.10^{2}\right)\end{array}$} & \multicolumn{7}{|c|}{$k_{\psi}\left(\mathrm{Nm} 10^{2}\right)$} \\
\cline { 2 - 7 } & 0.1 & 0.25 & 0.5 & 1 & 10 & 100 \\
\hline 0.1 & 18.0074 & 16.8436 & 16.4896 & 16.3186 & 16.1680 & 16.1531 \\
\hline 0.25 & 16.3225 & 15.1770 & 14.8333 & 14.6681 & 14.5231 & 14.5087 \\
\hline 0.5 & 15.6948 & 14.5701 & 14.2344 & 14.0732 & 13.9319 & 13.9179 \\
\hline 1 & 15.3710 & 14.2599 & 13.9290 & 13.7703 & 13.6312 & 13.6175 \\
\hline 10 & 15.0748 & 13.9779 & 13.6518 & 13.4956 & 13.3587 & 13.3452 \\
\hline 100 & 15.0449 & 13.9495 & 13.6240 & 13.4680 & 13.3313 & 13.3179 \\
\hline
\end{tabular}

TABLA 13

Parámetros Adimensionales de las Frecuencias NATURALES $\gamma_{1,2}^{2}, b / a=0.5$

\begin{tabular}{|c|c|c|c|c|c|c|}
\hline \multirow{2}{*}{$\begin{array}{c}k_{w}(\mathrm{~N} / \mathrm{m} \\
\left.10^{2}\right)\end{array}$} & \multicolumn{7}{|c|}{$k_{\psi}\left(\mathrm{Nm} 10^{2}\right)$} \\
\cline { 2 - 7 } & 0.1 & 0.25 & 0.5 & 1 & 10 & 100 \\
\hline 0.1 & 106.8751 & 104.2969 & 103.4922 & 103.0999 & 102.7523 & 102.7179 \\
\hline 0.25 & 99.1430 & 96.3793 & 95.5326 & 95.1225 & 94.7608 & 94.7250 \\
\hline 0.5 & 95.4513 & 92.6915 & 91.8542 & 91.4500 & 91.0943 & 91.0591 \\
\hline 1 & 93.3459 & 90.6157 & 89.7921 & 89.3952 & 89.0463 & 89.0118 \\
\hline 10 & 91.2980 & 88.6154 & 87.8102 & 87.4230 & 87.0829 & 87.0493 \\
\hline 100 & 91.0853 & 88.4086 & 87.6057 & 87.2196 & 86.8805 & 86.8471 \\
\hline
\end{tabular}

En las Tablas 14 y 15 se muestran los parámetros adimensionales de las frecuencias naturales $\gamma_{0,1}^{2}$ y $\gamma_{0,2}^{2}$ respectivamente para diferentes combinaciones de rigideces $k_{w}$ y $k_{\psi}$ en el disco anular con relación $b / a=0.7$.

TABLA 14

Parámetros Adimensionales de las Frecuencias Naturales $\gamma_{0,1}^{2}, b / a=0.7$

\begin{tabular}{|c|c|c|c|c|c|c|}
\hline \multirow{2}{*}{$\begin{array}{c}k_{w}(\mathrm{~N} / \mathrm{m} \\
\left.10^{2}\right)\end{array}$} & \multicolumn{7}{|c|}{$k_{\psi}\left(\mathrm{Nm} 10^{2}\right)$} \\
\cline { 2 - 7 } & 0.1 & 0.25 & 0.5 & 1 & 10 & 100 \\
\hline 0.1 & 51.0910 & 47.9903 & 47.0175 & 46.5422 & 46.1208 & 46.0789 \\
\hline 0.25 & 46.2711 & 43.1672 & 42.2090 & 41.7435 & 41.3322 & 41.2915 \\
\hline 0.5 & 44.1378 & 41.1009 & 40.1700 & 39.7189 & 39.3210 & 39.2816 \\
\hline 1 & 42.9617 & 39.9791 & 39.0683 & 38.6275 & 38.2388 & 38.2004 \\
\hline 10 & 41.8423 & 38.9224 & 38.0336 & 37.6039 & 37.2253 & 37.1878 \\
\hline 100 & 41.7273 & 38.8145 & 37.9280 & 37.4995 & 37.1220 & 37.0847 \\
\hline
\end{tabular}

TABLA 15

Parámetros Adimensionales de las Frecuencias NATURALES $\gamma_{0,2}^{2}, b / a=0.7$

\begin{tabular}{|c|c|c|c|c|c|c|}
\hline \multirow{2}{*}{$\begin{array}{c}k_{w}(\mathrm{~N} / \mathrm{m} \\
\left.10^{2}\right)\end{array}$} & \multicolumn{7}{|c|}{$k_{\psi}\left(\mathrm{Nm} 10^{2}\right)$} \\
\cline { 2 - 7 } & 0.1 & 0.25 & 0.5 & 1 & 10 & 100 \\
\hline 0.1 & 310.8493 & 304.7809 & 302.8570 & 301.9132 & 301.0741 & 300.9907 \\
\hline 0.25 & 288.7834 & 281.6257 & 279.3869 & 278.2942 & 277.3258 & 277.2297 \\
\hline 0.5 & 274.0642 & 266.5446 & 264.2217 & 263.0932 & 262.0959 & 261.9971 \\
\hline 1 & 264.0772 & 256.5091 & 254.1926 & 253.0708 & 252.0815 & 251.9836 \\
\hline 10 & 253.1951 & 245.7593 & 243.5058 & 242.4183 & 241.4612 & 241.3666 \\
\hline 100 & 252.0012 & 244.5913 & 242.3480 & 241.2658 & 240.3136 & 240.2195 \\
\hline
\end{tabular}

En las Tablas 16 y 17 se muestran los parámetros adimensionales de las frecuencias naturales $\gamma_{1,1}^{2}$ y $\gamma_{1,2}^{2}$ para diferentes combinaciones de rigideces $k_{w}$ y $k_{\psi}$ en el disco anular con relación $b / a=0.7$.

TABLA 16

Parámetros Adimensionales de las Frecuencias NATURALES $\gamma_{1,1}^{2}, b / a=0.7$

\begin{tabular}{|c|c|c|c|c|c|c|}
\hline \multirow{2}{*}{$\begin{array}{c}k_{w}(\mathrm{~N} / \mathrm{m} \\
\left.10^{2}\right)\end{array}$} & \multicolumn{6}{|c|}{$k_{\psi}\left(\mathrm{Nm} 10^{2}\right)$} \\
\cline { 2 - 7 } & 0.1 & 0.25 & 0.5 & 1 & 10 & 100 \\
\hline 0.1 & 52.3657 & 49.2823 & 48.3164 & 47.8448 & 47.4267 & 47.3852 \\
\hline 0.25 & 47.1591 & 44.0500 & 43.0920 & 42.6270 & 42.2163 & 42.1756 \\
\hline 0.5 & 44.8453 & 41.7996 & 40.8681 & 40.4171 & 40.0194 & 39.9801 \\
\hline 1 & 43.5698 & 40.5784 & 39.6670 & 39.2263 & 38.8380 & 38.7996 \\
\hline 10 & 42.3569 & 39.4293 & 38.5403 & 38.1110 & 37.7329 & 37.6956 \\
\hline 100 & 42.2324 & 39.3120 & 38.4255 & 37.9974 & 37.6205 & 37.5832 \\
\hline
\end{tabular}

TABLA 17

Parámetros Adimensionales de las Frecuencias NATURALES $\gamma_{1,2}^{2}, b / a=0.7$

\begin{tabular}{|c|c|c|c|c|c|c|}
\hline \multirow{2}{*}{$\begin{array}{c}k_{w}(\mathrm{~N} / \mathrm{m} \\
\left.10^{2}\right)\end{array}$} & \multicolumn{6}{|c|}{$k_{\psi}(\mathrm{Nm} \mathrm{10})$} \\
\cline { 2 - 7 } & 0.1 & 0.25 & 0.5 & 1 & 10 & 100 \\
\hline 0.1 & 312.5214 & 306.4748 & 304.5583 & 303.6183 & 302.7825 & 302.6994 \\
\hline 0.25 & 290.3662 & 283.2270 & 280.9945 & 279.9050 & 278.9395 & 278.8437 \\
\hline 0.5 & 275.5681 & 268.0656 & 265.7487 & 264.6233 & 263.6287 & 263.5302 \\
\hline 1 & 265.5225 & 257.9722 & 255.6620 & 254.5434 & 253.5570 & 253.4594 \\
\hline 10 & 254.5757 & 247.1602 & 244.9138 & 243.8299 & 242.8761 & 242.7818 \\
\hline 100 & 253.3748 & 245.9856 & 243.7496 & 242.6711 & 241.7222 & 241.6284 \\
\hline
\end{tabular}

En las Tablas 18 y 19 se muestran los parámetros adimensionales de las frecuencias naturales $\gamma_{0,1}^{2}$ y $\gamma_{0,2}^{2}$ respectivamente para diferentes combinaciones de rigideces $k_{w}$ y $k_{\psi}$ en el disco anular con relación $b / a=0.9$. 
TABLA 18

Parámetros Adimensionales de las Frecuencias NATURALES $\gamma_{0,1}^{2}, b / a=0.9$

\begin{tabular}{|c|c|c|c|c|c|c|}
\hline \multirow{2}{*}{$\begin{array}{c}k_{w}(\mathrm{~N} / \mathrm{m} \\
\left.10^{2}\right)\end{array}$} & 0.1 & 0.25 & 0.5 & 1 & 10 & 100 \\
\hline 0.1 & 532.1587 & 508.2088 & 500.5468 & 496.7749 & 493.4136 & 443.0792 \\
\hline 0.25 & 484.8360 & 458.0952 & 449.6172 & 445.4585 & 441.7611 & 441.3937 \\
\hline 0.5 & 448.3709 & 420.7466 & 412.0906 & 407.8633 & 404.1154 & 403.7435 \\
\hline 1 & 421.3481 & 393.9390 & 385.4351 & 381.2967 & 377.6355 & 377.2726 \\
\hline 10 & 390.2725 & 364.0459 & 355.9950 & 352.0911 & 348.6449 & 348.3038 \\
\hline 100 & 386.7941 & 360.7587 & 352.7749 & 348.9050 & 345.4894 & 345.1514 \\
\hline
\end{tabular}

TABLA 19

Parámetros Adimensionales de las Frecuencias Naturales $\gamma_{0,2}^{2}, b / a=0.9$

\begin{tabular}{|c|c|c|c|c|c|c|}
\hline \multirow{2}{*}{$\begin{array}{c}k_{w}(\mathrm{~N} / \mathrm{m} \\
\left.10^{2}\right)\end{array}$} & \multicolumn{6}{|c|}{$k_{\psi}\left(\mathrm{Nm} 10^{2}\right)$} \\
\cline { 2 - 7 } & 0.1 & 0.25 & 0.5 & 1 & 10 & 100 \\
\hline 0.1 & 2985.510 & 2943.721 & 2930.396 & 2923.843 & 2918.008 & 2917.427 \\
\hline 0.25 & 2875.318 & 2825.945 & 2810.231 & 2802.511 & 2795.640 & 2794.957 \\
\hline 0.5 & 2746.033 & 2688.779 & 2670.662 & 2661.781 & 2653.888 & 2653.104 \\
\hline 1 & 2600.243 & 2536.419 & 2516.437 & 2506.681 & 2498.034 & 2497.176 \\
\hline 10 & 2337.087 & 2270.240 & 2249.840 & 2239.971 & 2231.272 & 2230.411 \\
\hline 100 & 2299.366 & 2233.105 & 2212.958 & 2203.224 & 2194.651 & 2193.803 \\
\hline
\end{tabular}

En las Tablas 20 y 21 se muestran los parámetros adimensionales de las frecuencias naturales $\gamma_{1,1}^{2}$ y $\gamma_{1,2}^{2}$ para diferentes combinaciones de rigideces $k_{w} \mathrm{y} k_{\psi}$ en el disco anular con relación $b / a=0.9$.

TABLA 20

Parámetros Adimensionales de las Frecuencias NATURALES $\gamma_{1,1}^{2}, b / a=0.9$

\begin{tabular}{|c|c|c|c|c|c|c|}
\hline \multirow{2}{*}{$\begin{array}{c}k_{w}(\mathrm{~N} / \mathrm{m} \\
\left.10^{2}\right)\end{array}$} & 0.1 & 0.25 & 0.5 & 1 & 10 & 100 \\
\hline 0.1 & 533.6824 & 509.7825 & 502.1380 & 498.3750 & 495.0218 & 494.6883 \\
\hline 0.25 & 486.1540 & 459.4454 & 450.9789 & 446.8262 & 443.1343 & 442.7675 \\
\hline 0.5 & 449.4799 & 421.8731 & 413.2242 & 409.0006 & 405.2561 & 404.8846 \\
\hline 1 & 422.2805 & 394.8822 & 386.3835 & 382.2479 & 378.5894 & 378.2269 \\
\hline 10 & 390.9892 & 364.7731 & 356.7275 & 352.8266 & 349.3833 & 349.0424 \\
\hline 100 & 387.4864 & 361.4620 & 353.4837 & 349.6168 & 346.2042 & 345.8664 \\
\hline
\end{tabular}

TABLA 21

Parámetros Adimensionales de las Frecuencias NATURALES $\gamma_{1,2}^{2}, b / a=0.9$

\begin{tabular}{|c|c|c|c|c|c|c|}
\hline \multirow{2}{*}{$\begin{array}{c}k_{w}(\mathrm{~N} / \mathrm{m} \\
\left.10^{2}\right)\end{array}$} & \multicolumn{7}{|c|}{$k_{\psi}(\mathrm{Nm} \mathrm{10})$} \\
\cline { 2 - 7 } & 0.1 & 0.25 & 0.5 & 1 & 10 & 100 \\
\hline 0.1 & 2986.909 & 2945.134 & 2931.814 & 2925.263 & 2919.430 & 2918.850 \\
\hline 0.25 & 2876.713 & 2827.355 & 2811.647 & 2803.929 & 2797.060 & 2796.377 \\
\hline 0.5 & 2747.410 & 2690.172 & 2672.060 & 2663.181 & 2655.291 & 2654.507 \\
\hline 1 & 2601.583 & 2537.773 & 2517.796 & 2508.043 & 2499.398 & 2498.540 \\
\hline 10 & 2338.324 & 2271.492 & 2510.977 & 2241.231 & 2232.534 & 2231.674 \\
\hline 100 & 2300.586 & 2234.341 & 2214.199 & 2204.468 & 2195.898 & 2195.050 \\
\hline
\end{tabular}

Los resultados aquí mostrados, así como los de las referencias, fueron obtenidos utilizando un coeficiente de Poisson $v=1 / 3$, el cual es un valor típico para el acero. Se presentan en forma tabular para incluir diversas relaciones $b / a$, rigideces y modos de vibración.

En la Figura 3 se muestran las formas modales para $k_{w}=10000 \mathrm{~N} / \mathrm{m}$ y diferentes valores de la rigidez a flexión $k_{\psi}$ en el disco anular con relación $b / a=0.1$ en el modo de vibración $m=0, n=1$.

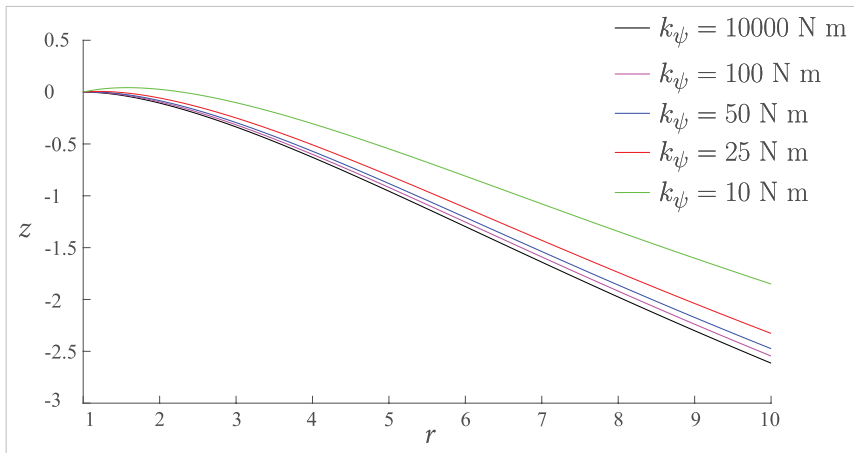

Figura 3. Formas modales del plato anular con soporte elástico para $k_{w}=10000 \mathrm{~N} / \mathrm{m}$, variando $k_{\psi}, m=0, n=1$.

En la Figura 4 se muestran las formas modales para $k_{\psi}=10000 \mathrm{~N} \mathrm{~m}$ y diferentes valores de la rigidez vertical $k_{w}$ en el disco anular con relación $b / a=0.1$ en el modo de vibración $m=0, n=1$. En la Figura 5 se muestran las formas modales para $k_{w}=10000 \mathrm{~N} / \mathrm{m}$ y diferentes valores de la rigidez a flexión $k_{\psi}$ en el disco anular con relación $b / a=0.1$ en el modo de vibración $m=0, n=2$.

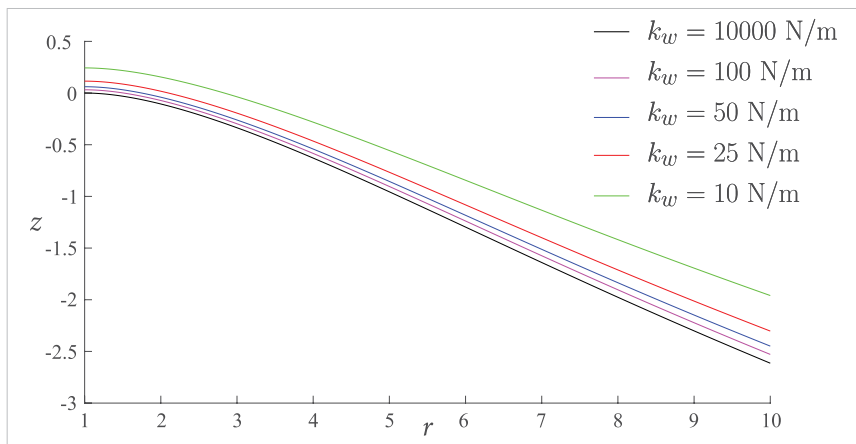

Figura 4. Formas modales del plato anular con soporte elástico para $k_{\psi}=10000 \mathrm{~N} \mathrm{~m}$, variando $k_{w}, m=0, n=1$. 


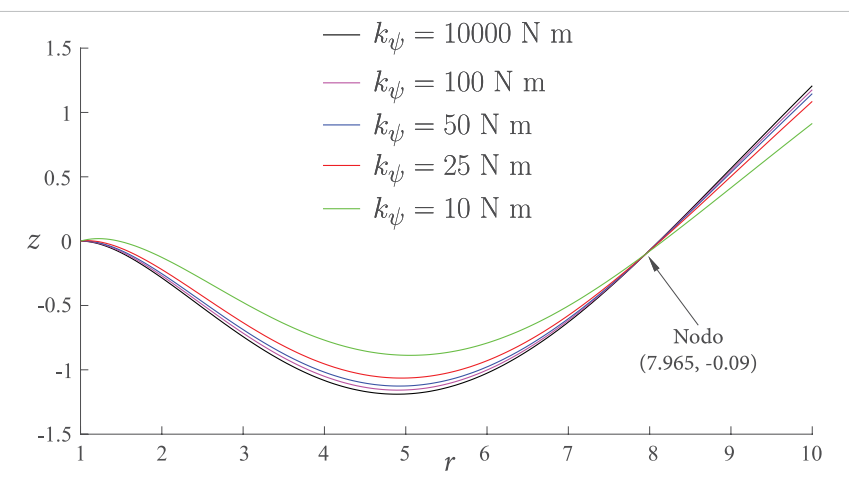

Figura 5. Formas modales del plato anular con soporte elástico para $k_{w}=10000 \mathrm{~N} / \mathrm{m}$, variando $k_{\psi}, m=0, n=2$.

En la Figura 6 se muestran las formas modales para $k_{\psi}=10000 \mathrm{~N}$ m y diferentes valores de la rigidez vertical $k_{w}$ en el disco anular con relación $b / a=0.1$ en el modo de vibración $m=0, n=2$.

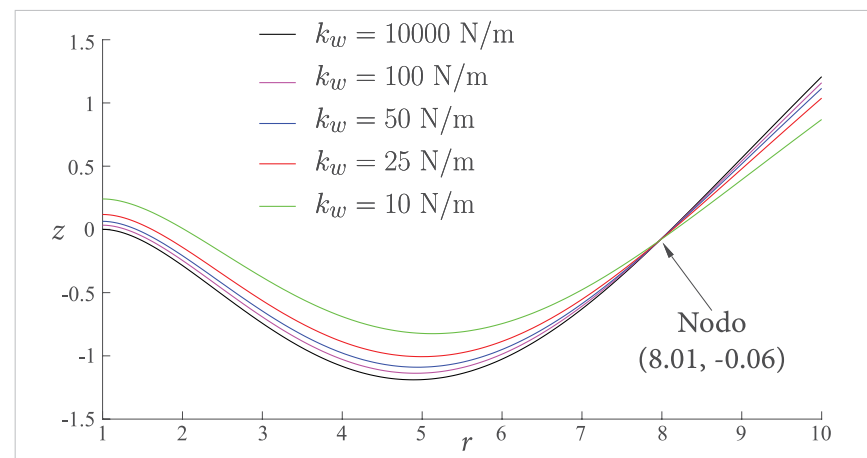

Figura 6. Formas modales del plato anular con soporte elástico para $k_{\psi}=10000 \mathrm{~N} \mathrm{~m}$, variando $k_{w}, m=0, n=2$.

\section{CONCLUSIONES}

Se presentaron los modelos de vibración libre de platos anulares delgados mediante la teoría de Kirchhoff para los soportes empotrado y elástico. Se obtuvieron los parámetros de frecuencia natural para $b / a=0.1,0.3,0.5$, 0.7 y 0.9 en ambos modelos. En todos los casos aquí analizados se encontró que la frecuencia fundamental de vibración corresponde al modo axisimétrico de vibración $m=0$.

En las Tablas 2 a la 21 se observa que las rigideces $k_{w}$ y $k_{\psi}$ tienen un efecto significativo en los parámetros $\gamma_{m, n}^{2} \mathrm{y}$ se puede distinguir que conforme los valores de las rigideces aumentan, los parámetros de frecuencia $\gamma_{m, n}^{2}$ disminuyen y se acercan a los valores del soporte empotrado que se muestran en la Tabla 1. También se advierte que los valores de $\gamma_{m, n}^{2}$ en general cambian más a lo largo de una misma columna que en una misma fila, es decir, que estos valores son más sensibles a los cambios en la rigidez $k_{w}$ que en la $k_{\psi}$ para los intervalos de valores presentados en este estudio. Los parámetros de frecuencias naturales aquí mostrados son una base para el análisis de vibraciones libres en discos anulares con sujeción elástica y son aplicables para discos con distintas dimensiones de radios interno y externo.

En las formas modales de las Figuras 3 y 5, donde se mantiene constante la rigidez $k_{w}$, se observa cómo la curvatura de estas se incrementa con respecto al incremento en la rigidez $k_{\psi}$, sin embargo, no se aprecia un desplazamiento en la ubicación del soporte $r=1$. Algo distinto ocurre en las formas modales de las Figuras 4 y 6 , donde se mantiene constante la rigidez $k_{\psi}$ y a medida que la rigidez $k_{w}$ disminuye, las formas modales tienden a desplazarse de la ubicación del soporte.

Para ambas modificaciones de rigideces en el modo $m=0, n=2$ de las Figuras 5 y 6 se observa la existencia de nodos en los cuales aparentemente ningún cambio en las rigideces tiene efecto. Las coordenadas de ambos nodos hacen suponer que su ubicación es aproximadamente la misma que la de los círculos modales, es decir, los valores donde $z=0$. Bajo esta premisa se puede decir que los círculos modales permanecen inalterados a cambios en las rigideces al menos para este tipo de discos anulares sin amortiguamiento.

\section{REFERENCIAS}

[1] K. L. Bowers y J. Lund, Eds., Computation and Control IV: Proceedings of the Fourth Bozeman Conference. Bozeman, Montana, EUA: Springer Science \& Business Media, 1994.

[2] J. N. Reddy, Theory and Analysis of Elastic Plates and Shells. Boca Raton, Florida: CRC Press, 2006.

[3] W. Weaver Jr., S. P. Timoshenko y D. H. Young, Vibration Problems in Engineering, 5. ${ }^{\mathrm{a}}$ ed. Nueva York: John Wiley \& Sons, 1990.

[4] C. Y. Wang, "Fundamental Frequency of a Circular Plate Supported by a Partial Elastic Foundation", J. Sound Vib., vol. 285, nos. 4-5, pp. 1203-1209, 6 ag. 2005.

[5] A. W. Leissa, Vibration of Plates. Washington, D.C.: NASA, 1969. 
[6] K. R. Chellapilla y L. B. Rao, "Vibrations of Elastically Restrained Circular Plates Resting on Partial Winkler Foundation", Open Acoust. J., vol. 2, no. 1, pp. 68-74, 2009. DOI: $10.2174 / 1874837600902010068$.

[7] C. Y. Wang y C. M. Wang, Structural Vibration: Exact Solutions for Strings, Membranes, Beams, and Plates. Boca Raton: Florida, CRC Press, 2013.

[8] P. A. A. Laura, R. H. Gutiérrez, H. C. Sanzi y G. Elvira, "The Lowest Axisymmetric Frequency of Vibration of a Circular Plate Partially Embedded in a Winkler Foundation", J. Sound Vib., vol. 185, no. 5, pp. 915-919, 1995. DOI: 10.1006/jsvi.1995.0429.

[9] R. Lal y R. Saini, “On Radially Symmetric Vibrations of Functionally Graded Non-Uniform Circular Plate Including Non-Linear Temperature Rise”, Eur. J. Mech., vol. 77, sept.-oct. 2019. DOI: 10.1016/j.euromechsol.2019.103796.

[10] L. B. Rao y C. K. Rao, "Vibrations of Circular Plates on Elastic Foundation with Internal Elastic Ring Support and Elastically Restrained Outer Edge", Vib. Eng.
Technol. Mach. (VETOMAC-IV), 2007, pp. 17-19. DOI: 10.13140/2.1.3931.6168.

[11] H. Zhang, R. Zhua, D. Shi y Q. Wang, "A Simplified Plate Theory for Vibration Analysis of Composite Laminated Sector, Annular and Circular Plate", Thin-Walled Struct., vol. 143, oct. 2019. DOI: 10.1016/j.tws.2019.106252.

[12] P. Hagedorn y A. DasGupta, Vibrations and Waves in Continuous Mechanical Systems. West Sussex PO19 8SQ, England: John Wiley \& Sons, 2007. DOI: $10.1002 / 9780470518434$.

[13] C. Y. Wang y C. M. Wang, Structural Vibration: Exact Solutions for Strings, Membranes, Beams, and Plates. Boca Raton, Florida: CRC Press, 2013.

[14] K. K. Żur, “Green's Function in Free Axisymmetric Vibration Analysis of Annular Thin Plates with Different Boundary Conditions", Int. J. Appl. Mech. Eng., vol. 20, no. 4, pp.939-951, 2015. DOI: 10.1515/ijame-2015-0060.

[15] R. D. Blevins, Formulas for Dynamics, Acoustics and Vibration. Chichester, West Sussex, Reino Unido: John Wiley \& Sons, 2015. 